\title{
Towards an Aesthetic Invariant for Graph Drawing
}

\author{
Jan Adamec and Jaroslav Nešetřil \\ Department of Applied Mathematics and Institute of Thoretical Computer sciences \\ (ITI) * \\ Charles University \\ Malostranské nám. 25, 11800 Praha, Czech Republic \\ nesetril@kam.ms.mff.cuni.cz
}

\begin{abstract}
In this paper we do not address the question of visualization, of picture processing of visual information. The information for us is already processed and ,typically, it is of a very simple type such as drawing (however not necessary a graph drawing). What we would like to answer is how to formalize the fact that such a picture (drawing) is harmonious. Harmonious we mean in the sense of aesthetic pleasing. We prefer the word harmonious to aesthetic (which is probably more in common usage) as an aesthetic feeling is probably highly individual and we cannot have an ambition to define (or even approach that). We propose an approach which should capture some features of a harmonious picture by means of the notion Hereditary Fractional Length (HFL). This approach is based on the analysis of curves [16] which in turn goes back to Steinhaus and Poincaré. The hereditary approach is based on the dual approach (it may be viewed as an approach dual to the Piaget's analysis of intelligence), 13. The Hereditary Fractional Length is preserved by scaling and rotations and it is a very robust parameter which can be computed for a large class of drawings and pictures. This is an important feature as a perception of harmony (and aesthetic pleasure) is a robust feeling. Perhaps this parameter could aid in the hierarchical approach to graph visualization and graph drawing in particular.
\end{abstract}

\section{Introduction}

What do have the following pictures same and what different? (The substance and the context are important artistic aspects. However we are interested in formal similarities of these pictures; one picture is a musical score - a sketch by Janáček 24, the other picture one of the Moduli - a sketch by Načeradský and the second author, [21.)

\footnotetext{
* Supported by a Grant LN00A56 of Czech Ministary of Education

P. Mutzel, M. Jünger, and S. Leipert (Eds.): GD 2001, LNCS 2265, pp. 287-296, 2002.

(C) Springer-Verlag Berlin Heidelberg 2002
} 

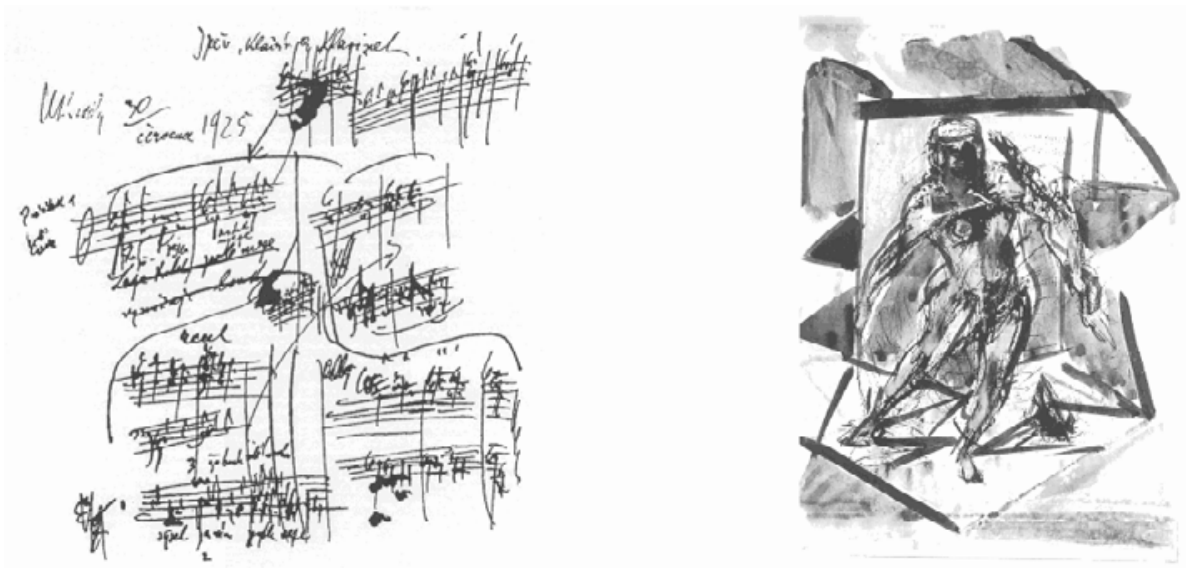

And, very simply, can we distinguish or order in some systematic way the following three figures (graph drawings using a program due to [8])?
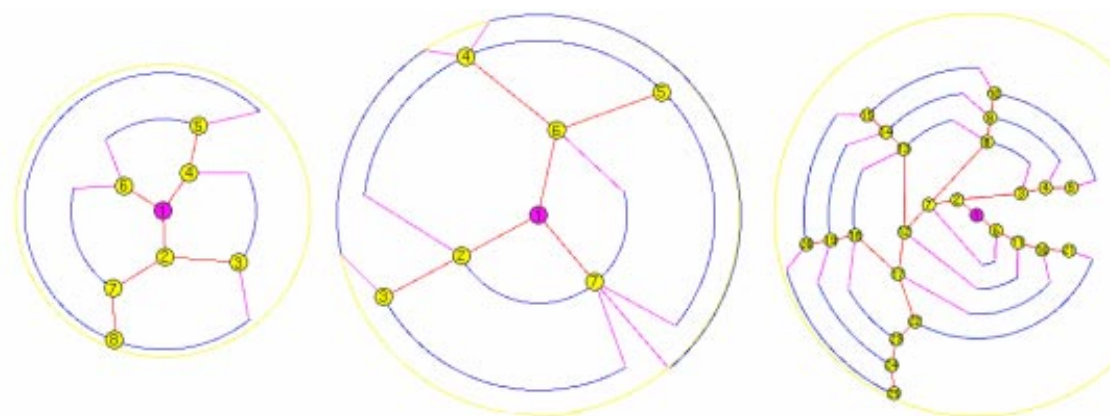

The modern version of these question is not how to teach a gifted and collaborating child what is nice and beautiful. Instead we need to teach an individual which is not collaborating at all and who takes every our information deadly seriously and exploits it to the last bit - a computer. People usually do not react this way (and if so, then only in comedies like 9 or [10; the fact that these great novels have a military setting is then not an accident). In order to "teach" a computer (and even without ambition for teaching, just dealing with it) we need a precision. And precision in the other words calls for some concrete measures or our phenomena, in the other words for invariants. The purpose of this note is to suggest such an invariant and to document some experiments which we performed.

\section{On Invariants}

The traditional principal problem of aesthetics (and art history) - to explain and to predict artistic and aesthetically pleasing took recently an unexpected twist. We do not explain and deal with individual instances, we have to classify 
a vast amount of data and we have to design procedures with likely harmonious output. This problem in its manifold variety is interesting already when our objects are well defined compositions composed from simple building blocks such as lines, squares, sticks,.... This in fact is a familiar exercise and training ground of schools of design and architecture and (traditional) art academies. This illustrates difficulty and variety of solutions even of simple situations. This should be not surprising if we realize how many simple lines needed, say Rembrandt or Picasso, to produce full images (for example drawings; 50 lines or even less!).

For our "simple composition from simple building blocks" we would like to create an invariant which would help us to categorize and order this compositions. It is difficult to say even on this simple level what it is an invariant, but we can certainly state which properties such an invariant should have:

i. invariant should be an (easy) computable aspect of the structure;

ii. invariant should be consistent (or invariant, meaning it should not change) under chosen modification of structure;

iii. invariant should be useful in that it can be used to catalogue, to order (which structure is "better"), to classify, to distinguish.

We propose here an invariant - called hereditary fractional length - to measure an aesthetic quality of a visual data (drawing, scheme, painting, note score, molecular data output and others). This invariant is presented in the next section.

\section{Hereditary Fractional Length of a Drawing}

Before defining the invariant we want to specify the rules under which the invariant should remain unchanged. Such rules were specified several times and are folklore in the visualization of scientific results. For example the book 4 lists (in section "Aesthetics") the following 11 graphic properties (called aesthetics which are commonly adopted:

Crossings, Area, Total Edge Length, Maximal Edge Length, Uniform Edge Length, Total Bends, Maximum Bends, Uniform Bends, Angular Resolution, Aspect Ration and Symmetry.

The names of these criteria are self-explanatory and together they form a very good paradigm for drawing of graphs. However most of them are specific for drawings of graphs (or structures like it) and they do not apply generally (for example to artistic drawings or sketches). Yet we believe that the aesthetic quality of visual algorithms should be tested on aesthetically charged objects. This is one of the underlying ideas of our approach.

There is another drawback of the above paradigm. In all of these criteria (with exception of angular resolution, where we want to maximize, and symmetry which is a structural property) we are aiming for a minimization (for example we want to minimize the total length of our drawing). That of course means that we have to optimize these criteria (as they are sometimes mutually pointed against each other) and we have to add to our paradigm preferences among them. 
However as optimization problems these criteria are computationally hard (see [4]).

Our approach is different and we believe it could add a new aspect to visualization and analysis of visual data.

Let us first specify objects which are relevant to our method:

A drawing $D$ is a finite set of curves in Euclidean plane. (As our drawings are man made we assume that the set is finite.) A curve is a continuous image of unit interval in plane (we mean a "nice" image; this is not place for technicalities).

A painting can be any image, including photos. It typically consists of differently colored areas.

An engraving is very special kind of object, since it can be included in the previous two classes. However seeing it as a drawing gives a lot more information.

For an infinite line $L$ denote by $i(L, D)$ the number of intersections of the line $L$ and drawing $D$. We define the fractional length $f \ell(D)$ of a drawing $D$ as the expected value of $i(L, D)$ where expectation relates to the random selection of line $L$. Fractional length could be also called Combinatorial Entropy from reasons to be mentioned later.

By virtue of this definition we note the following:

$i$. The fractional length $f \ell(D)$ is easily evaluated by a random generation of lines;

ii. $f \ell(D)$ is invariant under transposition and rotation;

iii. $f \ell(D)$ is invariant under scaling (i.e. "blowing up").

The role of randomness in the art has been discussed e.g. in [16, [17. These facts make it possible to evaluate (or very accurately to estimate) fractional length of many drawings schemata, drawings of artists (we systematically tested some of the early works of Picasso [22] and Kandinsky [12] as well some drawings from [21]). We also used the analytical description of tertiary structure of some of the proteins (provided by P. Pančoška, compare 11]). The software developed in [1] uses standard tools of digital image processing, see [2, [23] allows to handle very broad spectrum of examples. Most difficulties are with paintings, because they must be transformed into drawings in order to count number of intersections (several filters are applied on the picture to find its contours, resulting picture is drawing as we defined it before).

Let us state some specific examples: the above Janáček score had fractional length 18.55 which is quite similar to the fractional length 17.87 of the above drawing taken from [21. The three graph drawings depicted above had fractional lengths (from left to right) 5.70, 5.37, 6.96.

The method is flexible enough to handle complex drawings and pictures. For example classical prints from J. Verne novels when considered as a dense network of individual lines (no blurring). For example the following picture (due to Roux) [26] which serves as the front illustration of [14] has fractional length 136.6. 


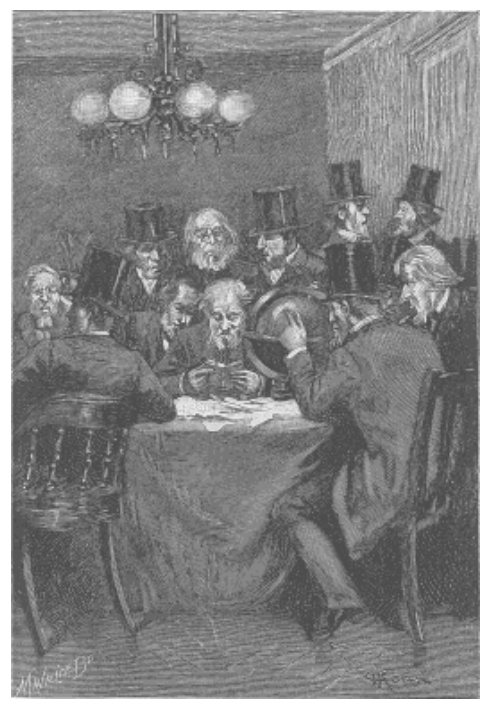

As expected the $n \times n$ lattice has approximate fractional length $n$. (As lattices are given analytically we can perform this experiment for very large $n$ ).

The following is a rare opportunity to compare a drawing (by Giacometti) and a related photography (which served as a basis of the drawing).

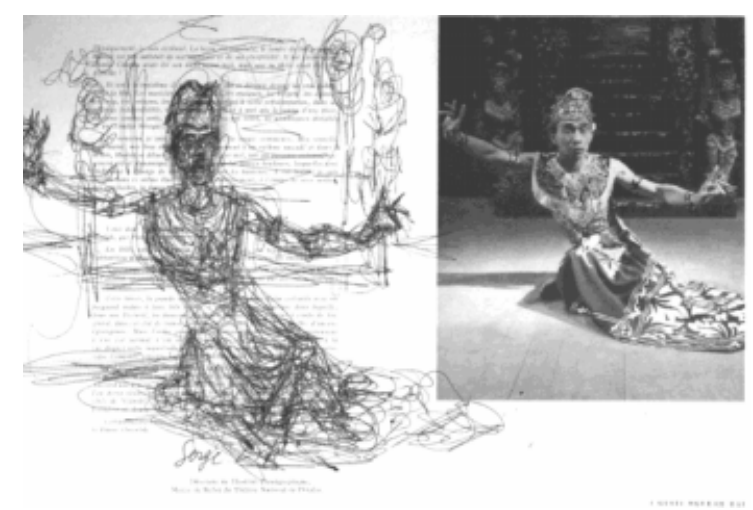

While the drawing has been scanned and the intersection numbers $i(D, T)$ were estimated directly the photo has been first processed by blurring and tracecontours filters. The fractional length of the photo is 14.43 , drawing is much more rich with approximate $f \ell(D) 26.37$.

Here the visual processing enriches the original design so that the drawing reduction has two times higher fractional length than the original taken as a drawing with variable heavy curves (29.64 vs 15.45$)$. 


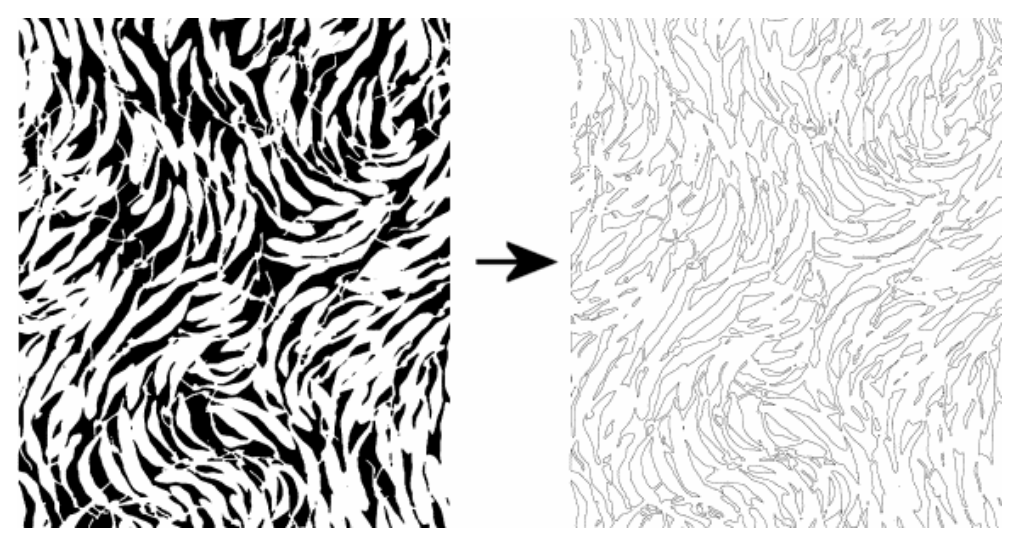

Here are several examples of the same scheme. The fractional lengths are as follows (from left to right): 24.06, 8.73, 17.86
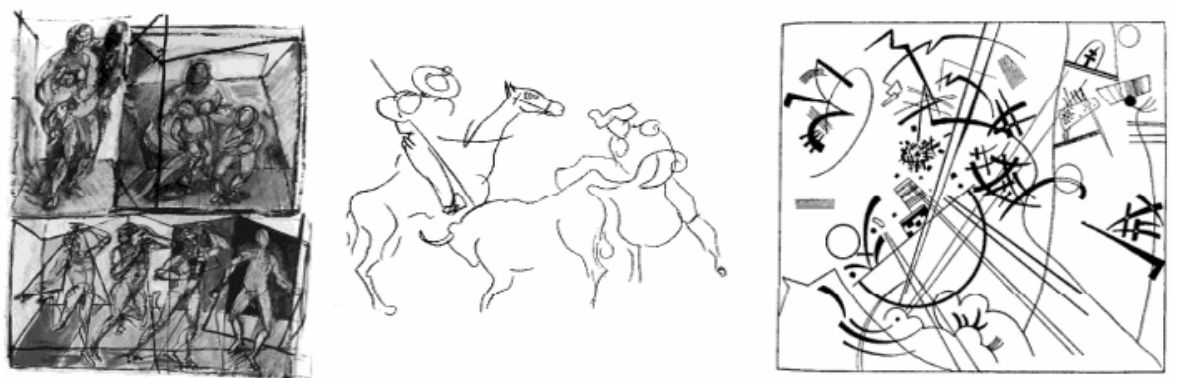

What do these numbers mean? What is a significance of the fractional length of a drawing? The definition of the fractional length is motivated by the research done by prof. Michel Mendes France in a series papers devoted to the analysis of curves, [16]. He defines the temperature $T(D)$ of a curve $D$ by the following formula

$$
T=\left(\log \frac{E(i(L, D))}{E(i(L, D))-1}\right)^{-1}
$$

where expectation relates to the random selection of the line $L$.

He related this parameter to entropy, dimension and other parameters which he defined in an analogy to fractal theory, statistical physics and geometric probability. These definitions rest on classical theorems due to Steinhaus [25] (which in turn goes to Poincaré). One can show that the $\log f l(D)$ is a good approximation of the entropy of a curve. Thus the name Combinatorial Entropy for the Fractional length.

By viewing a drawing as a set of curves and thinking of eulerian trail [14](in each component of the drawing) as a new curve of double length (as we traverse every segment of a drawing twice) we can define the the temperature by the same formula for a more general class of pictures (drawings). For large number of intersections (of a drawing $D$ with a line $L$ ) the temperature $T(D)$ is approximately equal to the average value of $i(D, L)-1=f \ell(D)-1$ while the 
entropy $H(D)$ is approximated by the fractional length $f \ell(D)$. It follows (and this is Steinhaus' theorem) that the fractional length $f \ell(D)$ of a drawing $D$ is approximated by the ratio

$$
2 \ell(D) / c(D)
$$

where $\ell(D)$ denotes the total length of the drawing $D$ and $c(D)$ denotes the length of circumference of the (convex closure) of $D$. The last formula motivates and justifies the name fractional length of our invariant: a drawing $D$ has its fractional length equal to $r$ iff the same (fractional) number of crossings is obtained when we traverse the circumference of $D$ by $r / 2$ times. As will be seen from the sequel we could not use the definition of either temperature or entropy as we demand that pure invariant has strong hereditary properties (see below). These properties (proportionality when considering a part of the picture) do not hold for neither temperature nor entropy.

Remark. We cannot resist the temptations to mention that similar ratios occur in art history. For example 3] is basing his esthetic theory on a ratio $M=C / O$ where $O$ denotes the "order" or "how many tricks are involved" and $C$ denotes the "complexity" which is proportional to "a preliminary effort necessary to perceive the object " and $O$ stands for "harmony, symmetry or order". Clearly this is meant symbolically and, as demonstrated in [3], the ratio has to be interpreted in individual cases. Our approach may be seen (on this symbolic level) as a statistical verification of these ideas. Motivated by the romantic symbolism of Marcel Duchamp this romantic algebra was used many more times with much less rigour, 7]. There is no romanticism in our formulas, these are exact results.

Based on these interpretations $f \ell(D)$ measures the information content and the amount of work which the artist (explicitly) put into his drawing. On the other hand side, by comparing various drawings of the same object (or theme) the lower $f \ell(D)$ indicates the elegance and simplicity of the output. However as such the fractional length captures only the global properties of the drawing (expressed by the total length of the drawing and the circumference). Still, via the average number of intersections it captures, implicitly, many properties of the drawing. In [13] we proposed a technique (based on the algebraical structure cogroup ) to generate harmonious objects. In our setting this can be formulated as follows:

\section{Hereditary Fractional Length Thesis}

A harmonious (or aesthetically pleasing) drawing or design has a fractional length in each of its (meaningful) parts proportional to the global fractional length. Here a meaningful part is a part which reflects the properties of D.A lower bound is obviously $\log i(D, L)$.

Clearly HFLT depends on the selection of (meaningful) parts - fields and as such it is only a program. HFL can be visualized as a matrix which has a hereditary structure (fields) where each field has a corresponding fractional 
length. There are at most $f \ell(D)$ fields and the depth of the hierarchical structure is at most $\log f \ell(D)$ (i.e. very efficient). How this is done is shown on the following examples.

We performed experiments with HFLT based on the regular subdivision of grids containing the circumference of our pictures and find some supporting evidence. Two particular results are included in this report. The Janáček score depicted above as one of the first illustrations of this paper gives results summarized by the following table (the middle of a rectangle indicates the fractional length of that rectangle).

\begin{tabular}{|c|c|c|c|c|c|c|c|c|c|c|c|c|c|c|c|}
\hline & & & & & 1,45 & $\mid 1,28$ & 1,03 & & & & & & & & \\
\hline & & 1.97 & 1,66 & & 1,47 & 11,75 & 1,02 & & & & & & & & \\
\hline & & 1,44 & 1,63 & & 1,49 & 2,50 & $1,1,4$ & & & & & & 1,20 & 2,63 & 1,61 \\
\hline & & 1,06 & 1,24 & $1,00^{2}$ & 2,16 & 2,53 & 2,55 & 1,34 & 1,03 & 1,97 & 1,11 & $\prod_{61,23}$ & 1,00 & 1,24 & 1,05 \\
\hline & & 1,54 & 2,00 & 1,03 & 1,89 & 2,37 & 1,81 & 1,38 & 1,11 & 2,62 & 1.89 & 2,72 & 1,59 & 2,43 & 1,53 \\
\hline 1,00 & 1,36 & & 1,64 & 1,01 & 2,28 & 2 & 2,08 & 1,63 & $\begin{array}{l}1,83 \\
-6\end{array}$ & 2,60 & 2,40 & 2,62 & 1,93 & 2,52 & 1,43 \\
\hline 1,32 & 1,64 & 1,00 & 2,11 & 1,36 & 3,24 & 2,44 & 1,98 & 1,39 & $1,76^{\circ}$ & 3,29 & 2,11 & 1,68 & 2,35 & 2,36 & 1,35 \\
\hline 1,55 & 1,83 & $1,00^{\circ}$ & 1,50 & 1,65 & 3,31 & 2,24 & 1,83 & 1,85 & 1,03 & 2,22 & 2,08 & 1.95 & 2,96 & 2,57 & 1,45 \\
\hline 1,20 & 1,83 & 2,70 & 1,38 & 1,67 & 1,21 & 1,32 & 1,94 & 3,29 & 1,74 & 1,43 & 1,01 & 1,73 & 2,43 & 2,69 & 1,48 \\
\hline 1.89 & 2,78 & 2,008 & 1,45 & 1,04 & $\begin{array}{r}1,75 \\
-5\end{array}$ & 2,41 & 2,26 & 3,04 & 1,62 & $2,01^{2}$ & 1,70 & 1,18 & 2,04 & 1199 & 1,07 \\
\hline 1,16 & 2,16 & 2,62 & 2,60 & 1,07 & $2,49^{\circ}$ & 2,11 & 2,02 & 2,36 & 1,34 & 1,97 & 2,75 & 1,39 & 2,14 & 1,01 & \\
\hline \multirow[t]{2}{*}{$1,00^{2}$} & 1,49 & $2,60^{4}$ & $\begin{array}{r}2,12 \\
\end{array}$ & 1,06 & 2,91 & 1,78 & 2,22 & 3,16 & 1,71 & $2,12^{4}$ & 2,90 & $T_{1,89}$ & 2,42 & 1,14 & \\
\hline & 1,35 & $\begin{array}{l}3,14 \\
\end{array}$ & 1,23 & 1,26 & 2.52 & 1,00 & 2,22 & 2,65 & 1,45 & $\begin{array}{r}1,73 \\
-4\end{array}$ & 2,67 & {$\left[\begin{array}{l}1,96 \\
-3\end{array}\right.$} & 2,37 & 1,01 & \\
\hline $1,05^{\circ}$ & 1,94 & 3,24 & 1,68 & 1,17 & 1,20 & 1,01 & 1,59 & 1,64 & 1,25 & 2,19 & 2,80 & 2,44 & 2,00 & & \\
\hline $\begin{array}{l}1,00 \\
-2,8 \\
\end{array}$ & 2,35 & 2,49 & 1,11 & & & & & & 1.14 & $\mid 2,37$ & 2,27 & 2,14 & 2,57 & $\mid 1,40$ & \\
\hline 1,00 & 2,04 & $1,50^{\circ}$ & 1,00 & & & & & & 1,01 & 1,17 & 1,52 & 1,36 & 1,04 & & \\
\hline
\end{tabular}

For the following drawing (of our institute in Prague)

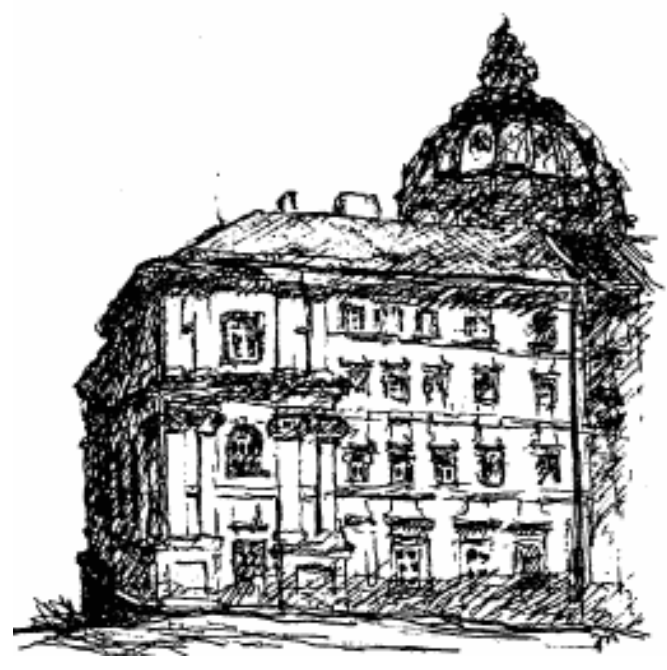

we found the following distribution of fractional lengths: 


\begin{tabular}{|c|c|c|c|c|c|c|c|c|c|c|c|c|c|c|c|}
\hline & & & & & & & & & & & & & & 1,72 & 2,10 \\
\hline & & & & & & & 1,00 & 1,02 & 1,76 & 1,38 & 1,68 & 1,23 & 1,23 & 1,50 & 2,77 \\
\hline & & & & & & 1,00 & 1,56 & 2,14 & 2,73 & 2,65 & 2,05 & 2,22 & 2,78 & 1,98 & 3,22 \\
\hline & & & & & & 2,30 & 2,75 & $3,13^{4}$ & 3,22 & 2.77 & 3,41 & 2.94 & 2,60 & 3,21 & 3,23 \\
\hline & 1,00 & & & 1,02 & 1,30 & 3,42 & 2,65 & 1,33 & 3,50 & 2,54 & 1,73 & 1,60 & 2,58 & 2,79 & 2,84 \\
\hline & & & & $1,00^{2}$ & 2,38 & 2,63 & 2,26 & 2,97 & 2,73 & 2,15 & 2,72 & $2,11^{4}$ & 2,72 & 3,14 & 2,53 \\
\hline & & & & 1,31 & 2,97 & 3,00 & 1,86 & 1,80 & 2,97 & 2,95 & 1,72 & 1,58 & 2,74 & 3,34 & 2,75 \\
\hline & & & & 1,11 & 2,07 & 2,95 & 2,64 & $\mid 1,75$ & 3,06 & 3,12 & 1,76 & 2,14 & 3,43 & 3,73 & 2,26 \\
\hline & & & & 1,12 & 2,54 & 2,64 & $2,80^{\circ}$ & 2,29 & 2,48 & 2,32 & 2,89 & 2,27 & 1,86 & 3,86 & 2,23 \\
\hline & & & 1,24 & 2,46 & 3,10 & 3,09 & 3,39 & $2,04^{4}$ & 2,83 & $2,38^{4}$ & 2,67 & $2,47^{4}$ & 2,96 & 3,92 & 2,56 \\
\hline & & 1,21 & 2.73 & 2.80 & 3.61 & 377 & 2.46 & 205 & $2.49^{\circ}$ & 2,14 & 2,55 & 2,11 & 1,77 & 3.66 & 286 \\
\hline $1,11^{2}$ & 2,12 & 2,31 & 2,98 & 2.82 & 3,44 & 3.44 & 2,93 & 2,24 & 2,80 & 2,16 & 2,70 & 2,22 & 1,97 & 3,63 & 2,86 \\
\hline 1,00 & 2,21 & 1,88 & 3,14 & 2,89 & 3,49 & 3,39 & 2,86 & 2,63 & 2,22 & 2,52 & 1,79 & $\left\{\begin{array}{l}2434 \\
2\end{array}\right.$ & 3.82 & 3,06 & 2,64 \\
\hline & & $\overline{1.22}^{4}$ & 3,06 & 2,81 & 2,45 & 2,38 & 2,95 & 2,62 & 2,65 & $2,86^{4}$ & 2,41 & 2,94 & 2,66 & 3.58 & 2,59 \\
\hline & & & 1,40 & 2,62 & 3,17 & 2,99 & 2,77 & 3,56 & $2,81^{\circ}$ & 2,74 & 3,42 & 2,49 & 1,34 & 2,21 & 2,89 \\
\hline & & & & & 1,22 & 2,24 & 1,98 & $1,33^{4}$ & 1,12 & 1,00 & & & & $1,00^{3}$ & 1,62 \\
\hline
\end{tabular}

\section{Conclusion}

Hereditary Fractional Length (or Hereditary Combinatorial Entropy) attempts to characterize harmonious and aesthetically pleasing pictures by a balanced distribution of Fractional Lengths (of Combinatorial Entropies) of their meaningful parts. One could also say that this measures not the simplicity (or complexity) of a picture but rather a relative balance and order of otherwise complex picture. This is perhaps in accord with our artistic experience and it has also an intuitive similarity with Birkhoff thesis mentioned above. It is pleasing to note that graphical objects as diverse as a musical score (i.e. Janáček sketch above) and an artistic drawing share a high degree of similarity thus perhaps pointing their similar (formal) aesthetic appeal.

Clearly many more examples have to be drawn but it seems that HFLT thesis is a good approximation to the notion of a harmonious drawing. But in any case an invariant is only one feature of otherwise very complicated phenomenon of visualization. But we believe that this, perhaps first, invariant for harmonious (i.e. aesthetic) drawing will inspire some further work in this direction.

\section{References}

1. J. Adamec: Kreslení grafü, diploma thesis, Charles University, Prague (2001).

2. G. A. Baxes: Digital Image Processing. Principles and Applications, Wiley, 1994.

3. G. D. Birkhoff: A Mathematical Theory of Aesthetics and its Application tp Poetry and Music, The Rice Institute Pamphlet, vol. XIX, 3 (1932), 342p.

4. G. DiBattista, P. Eades, R. Tamassia, I. G. Tollis: Graph Drawing. Algorithms for the Visualization of Graphs. Prentice Hall 1999.

5. H. Damisch: The Origins of Perspective, MIT Press 1994, original French edition Flamarion 1987. 
6. H. Damisch: Le travail de l'art: vers une topologie de la couleur? In: 21].

7. T. de Duve: Kant after Duchamp, MIT Press 1998.

8. H. de Fraysseix: A drawing software (personal communication).

9. J. Hašek: Osudy dobrého vojáka Švejka, 1920 (in English: The Good Soldier Schweik).

10. J. Heller: Catch-22, 1961

11. V. Janota, J. Nešetřil, P. Pančoška: Spectra Graphs and Proteins. Towards Understanding of Protein Folding. In: Contemporary Trends in Discrete Mathematics, AMS, DIMACS Series 49, 1999, pp. 237 - 255.

12. W. Kandinsky: Point and Line to Plane, Dover Publications 1979.

13. J. Kabele, J. Nešetřil: Remarks on Radically Different Aesthetic - a computational compromise (to appear).

14. J. Matoušek, J. Nešetřil: Invitation to Discrete Mathematics, Oxford Univ. Press 1998.

15. K. Mehlhorn, S. Naher: LEDA - A platform for combinatorial and geometric computing, Cambridge Univ. Press, 1999.

16. M. Mendès France: The Planck Constant of a Curve. In: Fractal Geometry and Analysis (J. Bélair, S. Dubuc, eds.) Kluwer Acad. Publ. 1991, pp. 325 - 366.

17. M. Mendès France, J. Nešetřil: Fragments of a Dialogue, KAM Series 95 - 303, Charles University Prague (a Czech translation in Atelier 1997).

18. J. Nešetrril: The Art of Drawing. In: Graph Drawing (ed. J. Kratochvíl), Springer Verlag,1999.

19. J. Nešetrril: Mathematics and Art, From The Logical Point of View, 2, 2/93 (1994), $50-72$.

20. J. Nešetřil: Aesthetics for Computers or How to Measure a Harmony (to appear in Visual Mind (ed. M. Emmer), MIT Press).

21. J. Načeradský, J. Nešetřil: Antropogeometrie I, II (Czech and English), Rabas Gallery, Rakovník, 1998 (ISBN 80-85868-25-3).

22. P. Picasso: Picasso - Der Zeichner 1893-1929, Diogenes, 1982.

23. W. K. Pratt: Digital Image Processing, Wiley, 1978.

24. M. Štědroň: Leoš Janáček and Music of 20. Century, Nauma, Brno, 1998 (in Czech).

25. H. Steinhaus: Length, shape and area, Colloq. Math. 3 (1954), 1 - 13.

26. J. Verne: Sans Dessus Dessous, J. Helzel (Paris), 1889. J. Nešetřil: Mathematics and Art, From The Logical Point of View, 2, 2/93 (1994), 50 - 72. 